\title{
The Line-Motion Illusion: Attention or Impletion?
}

\author{
Paul E. Downing and Anne M. Treisman \\ Princeton University
}

\begin{abstract}
When a brief lateral cue precedes an instantaneously presented horizontal line, observers report a sensation of motion in the line propagating from the cued end toward the uncued end. This illusion has been described as a measure of the facilitatory effects of a visual attention gradient (O. Hikosaka, S. Miyauchi, \& S. Shimojo, 1993a). Evidence in the present study favors, instead, an account in which the illusion is the result of an impletion process that fills in interpolated events after the cue and the line are linked as successive states of a single object in apparent motion.
\end{abstract}

Over the past 20 years or so, a large body of research has provided a picture of visual attention as a flexible, mobile source of processing facilitation that can be directed intentionally or summoned involuntarily by stimuli in the environment (Nakayama \& Mackeben, 1989; Posner, 1980; Yantis \& Jonides, 1984). One characteristic typically attributed to attention is its acceleration of the processing of visual stimuli. For example, in the paradigm developed by Posner (1980), participants make a speeded response to a target that can appear either to the left or to the right of fixation. When the target location is precued by a brief flash, participants are faster to respond than when the precue gives no advance information about the target location, even though fixation remains central. When the target occurs in the noncued location, responses are slowed. The difference in performance between these two conditions has been taken as a measure of the effects (both costs and benefits) of focal visual attention. Laberge (1983) found evidence for a gradient of attention, with the degree of facilitation decreasing as a function of distance from the center of the attended area.

Recently, another phenomenon has been proposed to demonstrate the facilitatory effects of attention (Hikosaka, Miyauchi, \& Shimojo, 1993a, 1993b; Miyauchi, Hikosaka, \& Shimojo, 1992; Shimojo, Miyauchi, \& Hikosaka, 1992). Hikosaka and his colleagues found that when a brief lateral cue precedes an instantaneously presented horizontal line, observers report that the line appears to unfold or propagate rapidly from its cued end to its uncued end (see Figure 1).

Portions of this work were included in a paper presented in May 1995 at the Annual Meeting of the Association for Research in Vision and Ophthalmology. This research was supported by a National Science Foundation (NSF) graduate research fellowship, U.S. Air Force Office of Scientific Research and Office of Naval Research Grant 90-0370, and NSF Grant SBR 95-11633.

We thank Kathy O'Craven for useful discussions and comments on earlier versions of the article.

Correspondence concerning this article should be addressed to Paul E. Downing or Anne M. Treisman, Department of Psychology, Princeton University, Princeton, New Jersey 08544. Electronic mail may be sent to Paul E. Downing at pdowning @ princeton.edu or to Anne M. Treisman at treisman@ princeton.edu.
The attentional explanation of this illusion given by Hikosaka et al. (1993a) is as follows: The initial cue summons attention to its location. Visual information processing is speeded at the cued location, with the degree of facilitation dropping off smoothly at increasingly greater distances from the cue. This, in turn, creates an asynchrony in neural responses. Motion detectors pick up the information from spatial regions close to the cued location earlier than from more distant locations, as they would if a stimulus were actually moving across the same locations. It is the firing of these motion detectors that is presumed to cause the illusory percept of motion along the length of the line.

We propose an alternative account of the line-motion illusion on the basis of an illusion observed in certain types of apparent motion displays. Classically, apparent motion is defined as an illusory impression of motion induced between two stimuli presented in succession or alternation at different locations. If the time interval and the distance between the two presentations are within the appropriate ranges, observers report seeing one object moving through space rather than seeing two independent perceptual events. Apparent motion is remarkably resilient to gross differences between the two stimuli (see, e.g., Kolers, 1972; Kolers \& von Grünau, 1976). For example, if the first stimulus $\left(S_{1}\right)$ is a small circle and the second stimulus $\left(\mathrm{S}_{2}\right)$ is a larger circle, observers report seeing a smooth increase in size occurring in the temporal interval between presentations. Similarly, if $S_{1}$ is a green square and $S_{2}$ is a red square, or if $S_{1}$ is a square and $S_{2}$ is a triangle, the visual system supplies the missing perceptual transformation. The observer in these cases sees an object changing color midway between the endpoints of its trajectory, or a smoothly changing shape. This filling-in process is known as impletion (see, e.g., Bundesen, Larsen, \& Farrell, 1983; Farrell \& Shepard, 1981; chapter 6 of Kanizsa, 1979; Orlansky, 1940; Shepard, 1984). One can argue that impletion reflects an implicit inference made by the visual system, which interprets ambiguous stimuli in terms of the most likely real-world state of affairs.

To apply this account to the line-motion illusion, we suggest that the visual system interprets the stimuli in the successive frames shown in Figure 1 as a single object traveling in apparent motion. This requires some account of 


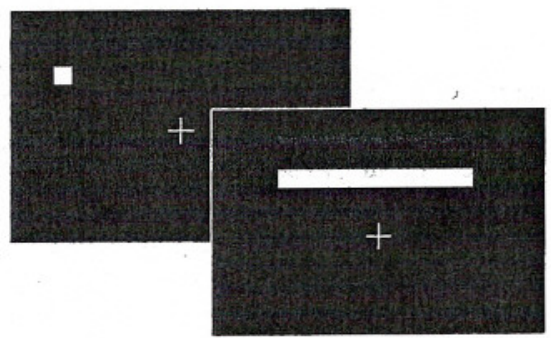

Figure 1. A schematic drawing of the line-motion illusion. The line is seen to unfold rapidly away from the precue.

the gross transformation in shape that the object undergoes. One possible account is that the dot (small square) jumps the minimal distance and then grows in length from there. We suggest that it is this impletion transformation that is seen as the illusory line motion, rather than the firing of motion detectors resulting from an attention-induced asynchrony. Orlansky (1940) noted a phenomenon similar to the illusion described here in a study of apparent motion. He compared the strength of apparent motion between rectangles of varying dimensions. In one condition, one stimulus was a square and the other was a narrow, elongated rectangle. Orlansky (1940) described the resulting impression as resembling the "opening and shutting of a camera bellows" (p. 26).

The crux of the difference between the two accounts of the line-motion illusion lies in the level of processing at which it is believed to arise. Where we emphasize higher level processes that ensure object continuity and coherence, Hikosaka et al. (1993a) have proposed a more bottom-up, sensory account. The present study is an attempt to separate these two accounts of the effect. Hikosaka et al. (1993a) made some arguments relevant to the approach we take here. We describe and address their arguments in the General Discussion.

\section{Experiment $1 \mathrm{~A}$}

In the process of exploring the basic line-motion illusion, we found that when the order of presentation of the dot and the line was reversed, an illusory shrinking motion was perceived. That is, the offset of the target line did not appear to be uniform but rather began at the uncued end and proceeded toward the cued end. To confirm our informal impressions, we showed naive participants displays in which the cue preceded, followed, or was simultaneous with the line.

\section{Method}

Participants. Participants in this and the following experiments were undergraduate students from Princeton University and the University of California at Berkeley. They received either course credit or monetary compensation ( $\$ 5$ for $1 \mathrm{hr}$ of participation). Sixteen participants participated in this initial experiment.

Apparatus and stimuli. An Apple Macintosh running MacProbe software Version 1.6 (Hunt, 1994), controlled stimulus presentation and response collection. Stimuli were presented on a 14 in. ( $235.6 \mathrm{~cm})$ Apple High Resolution Color Monitor, and responses were made on the standard Apple keyboard. The experiments were conducted in normal room lighting. Participants were asked to fixate on a given point, but their head position and eye movements were not monitored. Viewing distance was approximately $50 \mathrm{~cm}$. The same apparatus was used in all of the experiments.

All stimuli were white on a black background. The fixation stimulus was a cross subtending $0.6^{\circ}$ of visual angle. The cue was a square subtending $0.6^{\circ}$ of visual angle, and the target line subtended $0.6^{\circ}$ in height and $7.5^{\circ}$ in width. The cues were presented $5.5^{\circ}$ to either the left or the right of fixation, and $3.2^{\circ}$ above the fixation point, and the target line was centered $3.2^{\circ}$ above fixation.

Procedure. Participants were asked to report, for each display, whether it had led to a sensation of "shooting or unfolding motion within the line" and, if so, how strongly and in which direction. Each trial was initiated with the space bar, after which the fixation cross was presented and remained for the duration of the trial. Participants were instructed to keep their eyes on the cross throughout the trial. After each presentation, participants used a row of keys to indicate the direction and the strength of the motion perceived. The center key was marked with a zero to indicate no motion. On both sides of the center were four keys, marked with arrows to indicate very weak leftward (and rightward) motion through very strong leftward (and rightward) motion. Two additional keys were marked to indicate motion progressing outward from the center of the line and motion propagating inward toward the center. Thus, there were 11 possible response options on each trial.

Design. Cued side (left or right) was counterbalanced and randomly mixed across trials. Three presentation conditions were included. In the precue case, the cue was presented for $150 \mathrm{~ms}$, after which the target was presented (with an interstimulus interval of zero) for $150 \mathrm{~ms}$. The postcue case was simply the reverse of this; the target preceded the cue. Finally, on simultaneous trials, the cue and target were presented together for $150 \mathrm{~ms}$. This last condition was included, in part, to ensure that participants understood that "no motion" was a valid response and that they were willing to make such a response when that was what they perceived. Participants were given 36 randomly ordered trials, 12 in each cueing condition. ${ }^{1}$

\section{Results}

We coded participants' ratings on a 9-point scale, ranging from -4 for strong motion perceived as traveling toward the cue, through 0 for no perceived motion, to +4 for strong motion perceived as traveling away from the cue. Mean ratings were 2.40 for the precue condition $(p<.01), 0.03$ for the simultaneous cue condition ( $n s)$, and -1.20 for the postcue condition $(p<.01)$. In both the precue and postcue conditions, $100 \%$ of participants showed the effect in the direction of the mean.

The precue condition clearly replicates the finding of illusory motion reported by Hikosaka et al. (1993a). All participants reported seeing illusory motion in the line, in

\footnotetext{
${ }^{1}$ These participants were tested concurrently on other displays involving different hypotheses; however, pilot work with these displays run alone showed the same results.
} 
the direction away from the precued location. The postcue, however, produced an illusory shrinking motion toward the cue for all the participants. This follows naturally from the apparent motion impletion account. The sequence of events in both the precued and post-cued displays is interpreted as the trajectory of a single object. An impletion process interpolates the intermediate transformation of the moving object, resulting in an illusory percept of either growing or shrinking. We conducted an additional demonstration showing that both the growing and shrinking illusions can be produced in the same line, simply by alternating the cue and the line repeatedly at regular intervals.

It is unclear what should be predicted for these displays by the attention gradient hypothesis. On the one hand, we might suppose that attention is drawn to the postcue, prolonging the fading signal from the (now-absent) line, producing a gradient of fading from the far end of the line to the end near the cue. This would produce the percept our participants reported, in which the line's offset is seen to occur last at the point nearest the cue. On the other hand, it is equally plausible to suppose that attention might facilitate the offset signal generated by the line's disappearance, in much the same way that the onset is facilitated in Hikosaka et al.'s (1993a) account. Such a supposition would predict that the line would appear to shrink away from the cued location-the opposite of what we found. In other words, to account for these results by means of attention, we must assume that attention facilitates perception of visual onsets, and prolongs visual signals after an offset, but does not facilitate perception of visual offsets per se.

We have, in fact, tested the effect of attention on the perception of offsets in the Posner (1980) cueing paradigm (Treisman \& Downing, 1997). In that study participants were cued to one of two locations by the transient brightening of a box, after which a target event occurred. In one condition, the target was the offset of one of two points appearing in the cued or the uncued box at either side of fixation. In the other condition, the target was the onset of a point appearing in one of the same two locations. The cue signaled the target location on $67 \%$ of trials (valid) and signaled the other location on $22 \%$ (invalid). The task was to press a key as soon as the target event occurred; catch trials (11\% of trials), which required that no response be made, were included to ensure that participants were not merely responding to the cue. The validity effect (benefit of valid vs. invalid cue) averaged $31 \mathrm{~ms}$ for detection of onsets and $44 \mathrm{~ms}$ for detection of offsets. There was a significant main effect of validity, $F(1,7)=9.8, p<.05$, and no interaction $(F<1)$. Thus, attention facilitated the detection of offsets at least as much as detection of onsets. This result is the opposite of that required if the attention hypothesis is to account for the shrinking-line illusion when the cue follows the line. A postcue might be expected to have a weaker effect than the precue we tested, or even no effect, but there is no reason to suppose that it should reverse its effect. Thus, the alternate growing and shrinking of the line seems best explained as impletion induced by apparent motion.

\section{Experiment 1B}

If visual attention can be split between two disjoint spatial regions (a matter of some debate), then a display in which both ends of the line are precued should create a sensation of inward motion that meets at the center of the line (see Figure 2, top). Faubert and von Grünau (1992) reported just such a finding, which we replicate here. This result, however, is also consistent with an impletion account. It is known that apparent motion is readily seen to split or converge in displays for which there is no strict one-to-one mapping between successive stimuli (e.g., Kolers, 1972).

We can resolve the ambiguity between the two accounts with a display inspired by Ternus (1938). An additional line presented to one side of the double-cued display should, according to the apparent motion account, constrain the mapping of the objects in the two presentations (see Figure 2 , bottom). Specifically, the outer line should be assigned to its nearest cue, leaving the central line to match with the remaining cue. Motion should be produced in both lines, each in the same direction, away from the cue to which it was assigned. These predictions follow from the general tendency to make a one-to-one object mapping between successive stimuli when such a mapping is possible. Ac-
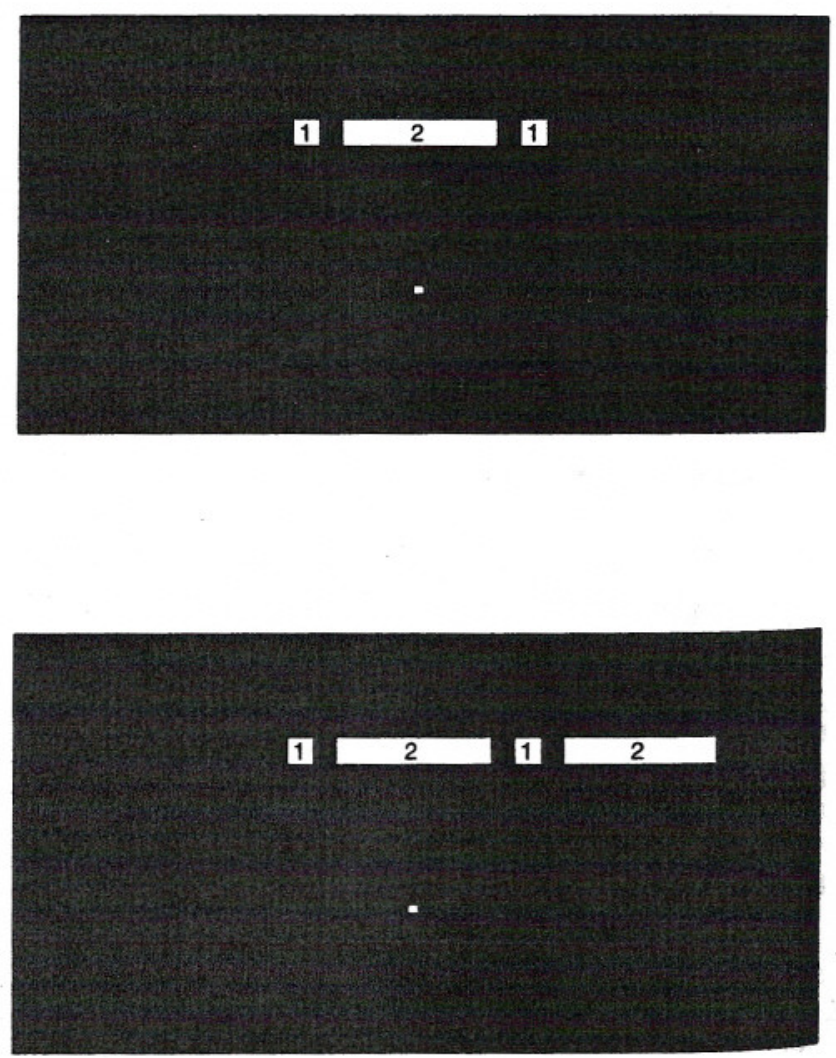

Figure 2. Illustration of the displays used in Experiment $1 \mathrm{~B}$. Numbers included here for diagrammatic purposes refer to the order of presentation. The top display generates an impression of inward motion. The second display, in which the precues are identical, produces lateral motion in both lines, toward the right. 
cording to the attention-gradient account, however, attention should be divided between the two initial cues. The motion in the central line should be seen to progress inward, whereas the motion in the outer line should be in the direction away from its single cue. (See also Faubert \& von Grünau, 1995, for a different account of similar displays, arrived at independently.)

\section{Method}

Participants. From the previously described pool, 10 new participants saw double-cued displays with a single line between the cues, and 10 others saw double-cued displays with two lines, one between the cues and one to the right or left of the cues.

Design. Each participant was given a total of 24 trials. For single-line participants, cue-target presentation order (precue or postcue) was counterbalanced. For double-line participants, the side on which the outer line was presented (left or right) and the cue-target presentation order (precue or postcue) were counterbalanced. The lines subtended $0.6^{\circ} \times 6.5^{\circ}$ visual angle, the dots were $0.6^{\circ}$ square, and the distance between the dots and the lines was $0.7^{\circ}$.

Procedure. Each trial began with a central fixation point. On precued trials, two cues were presented for $150 \mathrm{~ms}$. Immediately upon the offset of the cues, the line or lines appeared for $150 \mathrm{~ms}$. The stimuli appeared in the reverse order in the postcued condition. Single-line participants made motion ratings as in Experiment 1A. Double-line participants made two line motion ratings on each trial, reporting first for the left side of the display and then for the right.

\section{Results}

Participants who saw only a single line between two cues reported inward motion on $86 \%$ of precued trials. In contrast, the double-line group clearly interpreted the dualtarget displays as two objects jumping to one side and growing laterally; on $92 \%$ of precued trials, they judged the motion in both lines to be in the same direction, away from their associated cues. Postcued displays showed the same pattern in reverse: A single line was seen to disappear from the center outward, whereas dual lines were seen to shrink laterally, each toward just one of the cues.

Splitting attention cannot account for the results obtained from both types of double-cued line displays. If attention is responsible for the percept of inward motion seen in the single-line displays, then the same effect should have been obtained in the double-line displays. On the other hand, the impletion account explains how the assignment of perceptual events to objects constrains the manifestation of illusory line motion.

\section{Experiment 1C}

If our account is correct, apparent motion and the linemotion illusion should show the same effects of the temporal and spatial separation of the two stimuli. We tested this prediction by having participants make ratings both on line illusion displays and on comparable apparent motion displays, in which the line was replaced by a dot identical to the cue, located where the nearest end of the line would appear on the equivalent line trials. We varied both the temporal and the spatial separation between the two stimuli in both conditions.

We also addressed another question. It has been observed (Hikosaka et al., 1993a) that the line-motion illusion can be produced even when the initial cue remains present throughout the exposure of the line. Although these are not usually thought of as conditions that produce classical apparent motion, we decided to test the question directly: Would participants perceive apparent motion between two identical stimuli when the duration of the first stimulus completely overlapped that of the second? In all conditions of this experiment, the cue remained present throughout the presentation of the second stimulus.

\section{Method}

Participants. Eight new participants from the previously described pool participated in this experiment.

Design. Apparent motion displays and line-motion displays were presented in a blocked design. In the apparent motion block, we used four stimulus onset asynchronies (SOAs)-195 ms, 495 $\mathrm{ms}, 1,005 \mathrm{~ms}$, and $1,995 \mathrm{~ms}$ - and four possible distances between first and second stimulus: $1^{\circ}, 2^{\circ}, 3^{\circ}$, and $6^{\circ}$ of visual angle. The side of the first presentation (left or right) was counterbalanced. A total of 32 trials were presented in the block. The line-motion block was identical in design to the apparent motion block. The distance between the initial dot and the line was measured from the line's nearest endpoint.

Procedure. Each trial began with a fixation point presented alone for $1 \mathrm{~s}$. For the line-motion displays, a single dot was then presented, subtending $0.1^{\circ}$ of visual angle. After a varying delay, the line $\left(9.5^{\circ}\right.$ in length and $0.05^{\circ}$ in width) was presented, centered $3.5^{\circ}$ above fixation. The line's position was held constant for every trial; the eccentricity of the initial dot was varied to effect the distance manipulation. For the apparent motion displays, the procedure was the same, except that a single dot, identical to the initial one, was presented instead of a line, located in the same position as the end of the line nearest the cue. In both blocks, the initial dot remained present throughout the duration of the second stimulus, which was removed when the response was made.

Participants made ratings on apparent motion displays and linemotion displays on the same scale (and with the same instructions) as in the previous two experiments, with the exception that response options for inward and outward motion were not included. The instructions included a neutral description of the displays to be presented in each block, followed by an explanation that the experiment had to do with whether and how distance and time affected the perception of illusory motion. Half of the participants made ratings on apparent motion displays first, and half, on linemotion displays first.

\section{Results}

Means are shown in Figure 3A (distance effects), and 3B (timing effects). We performed an analysis of variance (ANOVA) on the data, with target type (dot or line), temporal interval $(195,495,1005$, or $1995 \mathrm{~ms})$, and distance $\left(1^{\circ}, 2^{\circ}, 3^{\circ}\right.$, or $\left.6^{\circ}\right)$ as variables. As expected, there was a reliable main effect of temporal interval, $F(3,21)=18.3$, 

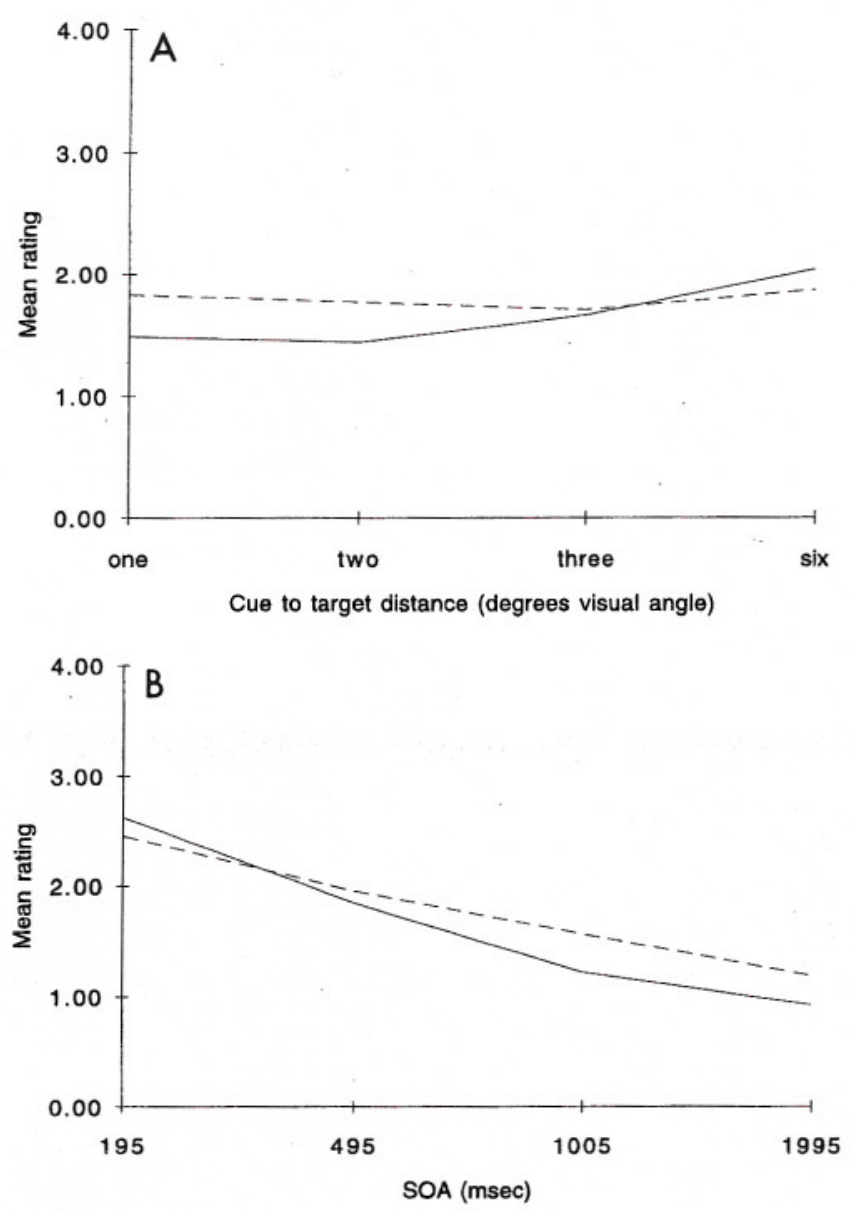

Figure 3. Similar effects of spatial (A) and temporal (B) separation on apparent motion and illusory line motion. Solid line indicates dot; broken line indicates line. SOA = stimulus onset asynchrony.

$p<.001$, with the mean rating dropping as the time between stimuli increased. The main effect of distance approached significance, $F(3,21)=2.6, p=.08$, as did the interaction of Temporal Interval $\times$ Distance, $F(9,63)=$ $1.9, p=.07$. Most important, there was no main effect of target type, nor did that variable interact with any of the others (all $F$ s $<1.61, n s$ ). It can be seen in Figure 3 that temporal and spatial parameters affected the line-motion illusion and apparent motion in very much the same way and that both persisted even though the first stimulus remained during the presentation of the second. Both illusions were relatively unaffected by the distance between the two stimuli, and a slight but significant motion effect persisted up to the longest SOA $(1,995 \mathrm{~ms})$, both for the line-motion condition and for the apparent motion control condition.

Most classical estimates of the maximum interval capable of supporting apparent motion refer to the interstimulus interval between successive stimuli. Because the cue in our experiment remained present throughout the trial, we varied only the SOA between cue and line. However, participants still reported apparent motion in all the same conditions and to the same degree as they saw in the line-motion condition. Before concluding with Hikosaka et al. (1993a) that an apparent motion account of the illusory line motion is ruled out in conditions involving long intervals and a cue that remains present, participants should be tested in an apparent motion control condition, with the same stimuli and the same forced-choice instructions as were used for the linemotion illusion. Even though we manipulated SOA instead of interstimulus interval, and used a rating scale that did not force participants to report motion, we still found directional effects in apparent motion at a 2-s SOA.

\section{Discussion}

The results presented thus far support the impletion hypothesis of the line-motion illusion. We found that postcues led to a complementary percept of illusory shrinking motion that is difficult to explain with a gradient model of attention. The effect of double cues on a central target line depended on whether an additional, peripheral line was also presented. If attention is assumed to be split by the two cues, it should enhance processing at those locations regardless of what follows. Finally, we found a close similarity in the effects of temporal and spatial parameters on apparent motion and line motion. In a study by Kawahara, Yokosawa, Nishida, and Sato (1995) on visual search for an "odd man out," manipulations of set size, contrast reversal (between the first and second stimulus), and interstimulus interval also all had nearly identical effects on search times for targets defined by apparent motion and targets defined by line motion, consistent with the idea that the two phenomena are closely related.

We explain these findings within a framework in which the cue and the target are linked, by means of apparent motion, as a single object. Consequently, illusory line motion then results from an impletion process that transforms the representation of that object to reconcile its two appearances.

\section{Experiment 2A}

Experiment 1 addressed the relationship between exogenously driven attention and the line-motion illusion. Hikosaka et al. (1993b) reported that the illusion also occurs under conditions in which attention is voluntarily directed rather than "pulled" by an exogenous cue. In their experiment, participants attentionally tracked one of four spots as they rotated around a circular path. When the rotation stopped, a line was presented connecting two of the spots. In the critical condition, one end of the line was at the original spatial location of the object that had been cued, and the other was at its final location, thus pitting object-based endogenous attention directly against location-based (spatial) attention. Participants uniformly reported line motion away from the object to which they had attended, regardless of how far it had rotated around the circle, for rotations of up to $360^{\circ}$. In Experiment 2, we attempted to measure directly the effects that were due to attention and those that 
were due to apparent motion and found a clear dissociation between the two.

We modified the procedure used by Hikosaka et al. (1993b) to include an objective measure of the allocation of attention (see Figure 4). Each trial began when one of four objects was cued by briefly flashing it off. Participants were instructed to maintain fixation and attentionally track the cued object until the rotation stopped. In the first block of trials, the task was a speeded discrimination, in which participants were to identify a target letter ( $\mathrm{T}$ or $\mathrm{X}$ ) that always appeared at the final location of the cued item. In the second block, letter discrimination trials were intermixed randomly with line-motion trials. In contrast to Hikosaka et al. (1993b), we presented the lines at an orientation orthogonal to the direction of rotation of the display (i.e., as a diagonal joining two opposite dots rather than as a line joining two adjacent dots). For half of the trials, the line was oriented such that one of its endpoints abutted the final location of the cued spot. According to the attentional account, this should have evoked illusory line motion away from that location. For the other half of the trials, the line was oriented at right angles to the line that abutted the cued spot, so that neither end of the line was adjacent to the cued
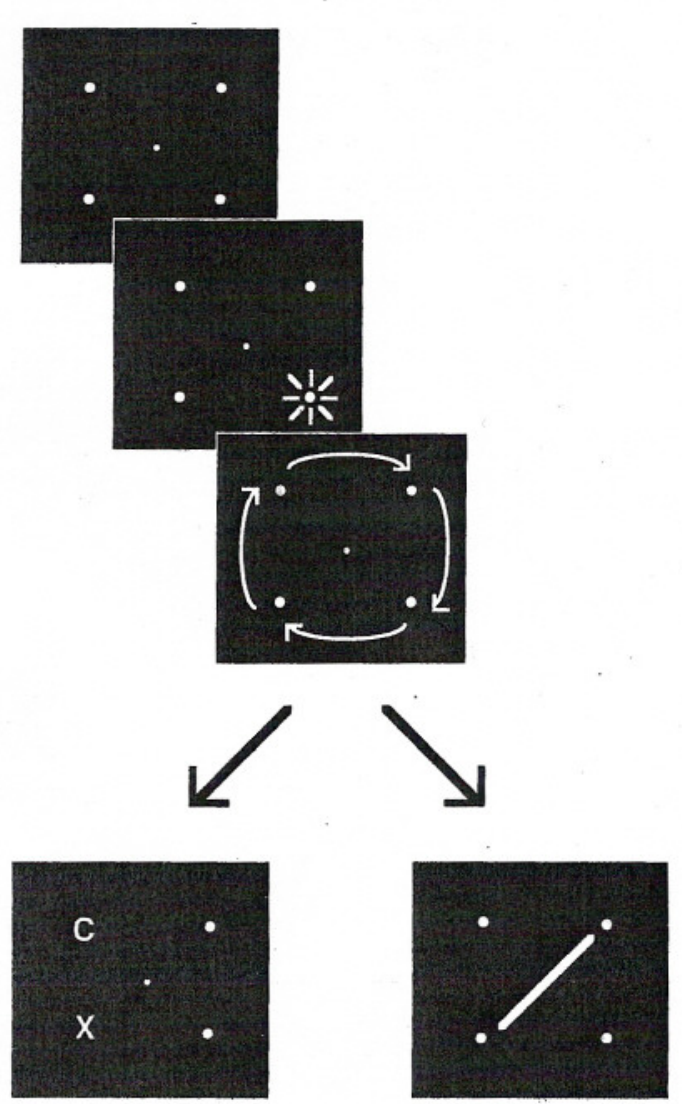

Figure 4. A schematic of the displays used in Experiment 2A. Participants track one of four rotating spots with attention, then either make line-motion judgments or speeded letter discriminations. The same displays were used in Experiment 2B except that the line was replaced with a central dot identical to the other four. object. These trials were included to reduce the likelihood that participants would become aware of a systematic relationship between the cued spot and the line.

In the final block of the experiment, participants once again exclusively performed the letter discrimination task. A small proportion of invalid trials was included for the first time in this block, to give an independent assessment of the allocation of participants' attention to the cued object. To the extent that they were slower to make discrimination judgments when the target letter appeared at an uncued object, we can infer that they were attending as instructed.

A final concern was that participants, even if they saw very little illusory motion, might feel compelled to use the full range of possible responses on the scale. To counter this, we included a condition in which, on both accounts, a strong illusory line motion would be expected: On some trials, the line was presented immediately after the to-beattended item was flashed, and the spots did not rotate at all. Thus, the two events (offset of cue and onset of line) occurred within a time interval suitable for obtaining apparent motion. According to both the attentional and the apparent motion accounts, this condition was expected to elicit illusory line motion.

\section{Method}

Participants. Twenty-four new participants from the previously described pool took part in this experiment.

Stimuli. The displays were constructed to match those of Hikosaka et al. (1993b), with the exception of the orientation of the lines. The four spots, colored white, were located on the corners of an imaginary square, centered at fixation, $3.6^{\circ}$ on a side. Each spot subtended $0.35^{\circ}$ of visual angle on a side; the fixation was $0.15^{\circ}$. The spots rotated in synchrony at a rate of $60^{\circ}$ per second around an imaginary circle having a radius of $2.5^{\circ}$. The letters used in the experiment ( $\mathrm{T}$ and $\mathrm{X}$ for targets, $\mathrm{C}$ and $\mathrm{S}$ for distractors) were presented in white and subtended approximately $0.8^{\circ} \times 0.6^{\circ}$ of visual angle. The target line subtended $3.8^{\circ}$ in length and $0.35^{\circ}$ in width and was oriented at an angle of $43^{\circ}$ from the horizontal.

Design. The experiment consisted of three blocks. The first block contained 24 letter discrimination trials. The letters were presented equally often after each of three angles of rotation $\left(0^{\circ}\right.$, $90^{\circ}$, or $180^{\circ}$ ). Cued object (top left, top right, bottom left, or bottom right) and direction of rotation (clockwise or counterclockwise) were counterbalanced. The position of the distractor letter was chosen randomly on each trial from the two locations that were neither the initial nor the final position of the cued object.

The second block consisted of a replication of the first, intermixed randomly with 48 line-judgment trials, for a total of 72 trials in the block. The same variables were manipulated for the linejudgment trials as for the letter task, with the additional variable of line orientation (either abutting the final position of the cued object or orthogonal to that).

The final block consisted of two replications of the first block, in addition to 18 invalid trials, giving a total of 66 trials in this block, $27 \%$ of them invalid. Invalid trials consisted of three replications of each possible rotation direction (clockwise or counterclockwise) at each angle of rotation $\left(0^{\circ}, 90^{\circ}\right.$, or $\left.180^{\circ}\right)$. The location of the target in the invalid trials was chosen randomly under the same constraints as those applied to the distractor item on valid trials.

Procedure. After the participant initiated each trial with the 
space bar, a fixation point was presented, remaining for the duration of the trial. The four spots were then presented, remaining for $675 \mathrm{~ms}$ before one of them was cued. The cue was given by flashing the object off for $300 \mathrm{~ms}$, after which there was a pause of $75 \mathrm{~ms}$ during which it was again visible. ${ }^{2}$ In the $0^{\circ}$ rotation condition, the letters (or line) were then immediately presented for a duration of $60 \mathrm{~ms}$. In the other two conditions, the spots rotated $90^{\circ}$ or $180^{\circ}$, followed by the presentation of the letters or line for $60 \mathrm{~ms}$.

Participants were instructed to maintain fixation throughout the trial, and to attend continuously to the flashed object. They were informed of the predictive relationship between the cue and the eventual location of the target letter; in addition they were informed that this contingency was less reliable in the final block. Responses were collected as in Experiment 1A with the exception that the inward and outward responses were not included. As the target lines were angled, participants were instructed to use the rightward response keys if the net direction of the motion within the line was to the right, and vice versa for the left. Participants first received 12 randomly ordered practice trials drawn equally from the line-judgment and letter discrimination tasks. At the beginning of each block, a reminder was provided on the screen about the types of trials that it would contain.

\section{Results}

Looking first at trials testing the efficiency of selective attention (from the third block only), we performed an ANOVA with cue validity (valid or invalid) and extent of rotation $\left(0^{\circ}, 90^{\circ}\right.$, or $\left.180^{\circ}\right)$ as the variables (see Table 1$)$. We found a significant main effect of cue validity on reaction time: Participants responded more rapidly on valid trials than on invalid trials, $F(1,23)=10.3, p<.005$. This is evidence that they were in fact attending to the cued object. In addition, there was a significant main effect of extent of rotation, $F(2,23)=6.9, p<.005$. Participants were fastest to respond in the $0^{\circ}$ rotation condition, slower in the $90^{\circ}$ condition, and slowest in the $180^{\circ}$ rotation condition. It is important to note that these two variables did not interact, $F(2,46)<1, n s$. The size of the validity effect thus did not depend on the extent of rotation of the objects in the display. Participants appear to have attended to the cued object throughout each trial.

The results of the line motion judgments are given in Table 2. An ANOVA with degree of rotation $\left(0^{\circ}, 90^{\circ}\right.$, or $\left.180^{\circ}\right)$ showed a significant effect of rotation, $F(2,46)=$ 23.9, $p<.001$ : Illusory motion was much stronger in the $0^{\circ}$ rotation than after the spots had rotated $90^{\circ}$ or $180^{\circ}$. Individual $t$ tests conducted on each condition showed that ratings were nonzero in all three conditions, suggesting that

Table 1

Effects of Cue Validity on Discrimination Reaction Time (in Milliseconds) in Experiments (Exp.) $2 A$ and $2 B$

\begin{tabular}{cccccccccc}
\hline & \multicolumn{2}{c}{$0^{\circ}$ rotation } & & \multicolumn{2}{c}{$90^{\circ}$ rotation } & & \multicolumn{2}{c}{$180^{\circ}$ rotation } \\
\cline { 2 - 3 } Exp. & Valid & Invalid & & Valid & Invalid & & Valid & Invalid \\
\hline 2A & 528 & 568 & & 574 & 608 & & 592 & 667 \\
2B & 516 & 532 & & 559 & 574 & & 573 & 617 \\
\hline
\end{tabular}

Table 2

Mean Motion Ratings and Percentage of Participants Seeing Some Motion in the Direction Away From the Cued Object in Experiments (Exp.) $2 A$ and $2 B$

\begin{tabular}{cccc}
\hline Measure & $0^{\circ}$ rotation & $90^{\circ}$ rotation & $180^{\circ}$ rotation \\
\hline Exp. 2A & & & \\
Motion rating & $2.00^{* *}$ & $0.64^{* *}$ & $0.73 * *$ \\
$\%$ participants & 96 & 71 & 79 \\
Exp. 2B & & & \\
Motion rating & $2.80^{* *}$ & $0.66^{* *}$ & $0.44 \dagger$ \\
$\%$ participants & 100 & 88 & 75 \\
\hline
\end{tabular}

Note. The motion rating scale ranged from 0 to 4 ; positive values indicate motion away from attended cue.

$\dagger p<.10 . \quad * * p<.01$.

a line-motion illusion might indeed be induced by endogenous attention alone.

However, there is an alternative account: Rather than inducing a neural asynchrony that stimulates motion detectors, attention may bias the apparent motion binding process that selects which stimuli represent the same object across time. We suggest that when the target line suddenly appears, a retrieval process seeks to link it to a previously existing object (Kahneman, Treisman, \& Gibbs, 1992). Proximity is one variable that affects the choice. By this criterion, there are two equally likely candidates in the rotating spot displays: the cued spot and the equidistant spot opposite it. Another criterion biasing the retrieval process may be attention: When an observer attends to one of the two adjacent spots, as in the present experiment, the attended spot may receive precedence in the binding process, making it more likely to be bound to the line than the other spot. The impletion process then gives rise to the illusory growth of the line away from the attended spot. The role of attention, in this account, is not to induce a gradient of facilitation that results in an input to motion detectors, but to bias the choice of objects to be bound across temporal and spatial intervals. One prediction from this account is that if the line is replaced with a single, central spot, apparent motion will tend to be seen as directed from the attended spot to the central spot. The following experiment tested this prediction.

\section{Experiment 2B}

This experiment was designed to determine the effects of attention on the perception of apparent motion in ambiguous displays, in which more than one mapping between successive frames is possible.

\section{Method}

Participants. Eight new participants from the previously described pool participated in this experiment.

\footnotetext{
${ }^{2}$ Results of a pilot study showed that apparent motion is seen under similar conditions, namely, when the first stimulus is blinked off, replaced, and then followed at a brief interval by a second adjacent stimulus.
} 
Stimuli. The stimuli were identical to those in Experiment 2A, except that the line was replaced with a central dot identical to the four rotating dots.

Design. The design was similar to that of Experiment 2A. Again, three blocks were used, counterbalanced on the same factors and with the same number of trials in each. Participants received 40 practice trials, consisting of 12 letter discrimination trials followed by a random mixture of 16 dot trials and 12 letter trials.

Procedure. The procedure was identical to that of Experiment 2A. Participants reported their impressions of apparent motion for the target spot on the same scale used previously, pressing one of the right keyboard keys if the direction of motion of the dot was predominantly to the right, one of the left keyboard keys if it was predominantly to the left, and the center key if they saw no motion.

\section{Results}

The results of the letter discrimination task are shown in Table 1. A main effect of validity was found, $F(1,7)=$ $12.8, p<.01$, with responses on invalid trials being slower than on valid trials. The main effect of extent of rotation was also significant, $F(2,14)=5.5, p<.05$. Responses were fastest in the $0^{\circ}$ rotation condition and slowest in the $180^{\circ}$ condition. As in Experiment 2A, these variables did not interact, $F(2,14)=0.43$, ns.

The results of the apparent motion judgments are shown in Table 2. A one-way ANOVA with degree of rotation $\left(0^{\circ}\right.$, $90^{\circ}$, and $180^{\circ}$ ) showed a significant main effect of rotation, $F(2,14)=33.8, p<.001$. The $0^{\circ}$ rotation trials generated a strong impression of apparent motion between the flashed cue and the subsequent target spot. Although the strongest motion ratings came from the $0^{\circ}$ rotation condition, as expected, participants did show a tendency to report apparent motion in the $90^{\circ}$ and $180^{\circ}$ rotation conditions as well. An ANOVA comparing Experiments 2A and 2B revealed an interaction between experiment and extent of rotation, $F(2,60)=3.7, p<.05$. Simple effects showed that this interaction can be attributed to stronger ratings of motion for dot targets than line targets at $0^{\circ}$ rotation, $F(1,30)=3.3$, $p=.08$. Rating strength did not differ between the two target types at $90^{\circ}$ or $180^{\circ}$ rotation (both $F \mathrm{~s}<1$ ).

Directly comparing ratings between the two studies is not ideal, as participants were rating qualitatively different displays. We can better compare the two studies by looking at how many participants show an overall tendency to report motion away from the cued object, as a function of the extent of rotation. These proportions are shown in Table 2, and it can be seen that they are quite similar across the two studies. We take this result to support the account outlined above: When faced with an ambiguous mapping between successively appearing stimuli, new onsets will be bound more often to previously attended objects than to unattended objects. Note that there is no ready explanation for why a gradient of attention should affect apparent motion the way it did in this experiment. The central target had negligible spatial extent and was equidistant from the attended spot and the spot diagonally opposite. Moreover, attention measured by the cue validity effect on letter discrimination was at least as strong after the $180^{\circ}$ rotation as at $0^{\circ}$, whereas ratings of illusory line motion and apparent motion both declined to a fraction of their initial strength.

\section{Experiment 3}

In another test of whether endogenous attention alone can produce illusory line motion, Hikosaka et al. (1993b) presented a horizontal line immediately after two peripheral cues, one red and one green. Observers were instructed to attend, without moving their eyes, to one of the two colored cues. Hikosaka et al. reported that this manipulation produced illusory line motion similar to that found with exogenous cues. The only difference was that it took longer to develop: The cues had to precede the target by at least 300 to $400 \mathrm{~ms}$ to generate the effect, compared with less than $100 \mathrm{~ms}$ with exogenous cues.

We replicated and extended Hikosaka et al.'s (1993b) procedure, adding a check on the effectiveness of attention similar to that used in Experiment 2. Participants again performed a letter discrimination task, in which one of the colored cues would always predict the location of the subsequent target letter.

Pilot studies we conducted (and Experiment 1B) suggested that participants would generally report inward motion for double-cued displays such as those described above. The question, then, was whether the allocation of attention to just one of the cues would bias the percept, making the motion appear stronger from the cued side. Participants were asked first to report, for each display, whether they saw lateral motion, inward motion, or no motion within the line. On trials for which participants reported motion, they were asked to describe their percept more precisely. For lateral motion trials, participants rated the direction and strength of the motion percept. For inward motion trials, participants reported, using the same rating scale, whether the motion was equally strong from both directions or biased in one direction or another.

A third and final display type was included to test whether ambiguous apparent motion would be biased by attention. The logic here was the same as that in Experiment 2B. We included trials identical to the line trials, except that the line was replaced by a single central spot. As with the line, this configuration typically produces an impression of converging, inward motion. The question was whether attention would affect the line-motion and apparent motion displays in the same manner by biasing the direction of perceived inward motion away from the attended side.

\section{Method}

Participants. Eight undergraduates from the pool described above participated in this experiment.

Stimuli. The colored cues were red and green squares, each subtending $0.6^{\circ}$ of visual angle on a side. The target line was white, subtending $7.5^{\circ} \times 0.6^{\circ}$. Cues were presented $5^{\circ}$ to the left and right of fixation, and $1.6^{\circ}$ above it; the letters were presented at the same locations as the cues. The letters used in the discrimination task were $\mathrm{T}, \mathrm{X}, \mathrm{S}$, and $\mathrm{C}$; they subtended approximately $0.9^{\circ}$ of visual angle and were colored white. 
Design. The experiment consisted of four blocks. The first consisted only of the letter discrimination task. Target letter (X or $\mathrm{T}$ ), nontarget letter (C or S), and number of cues (valid cue only or both cues) were manipulated orthogonally. To assess the possible effects of eye movements, and the slower deployment of endogenous attention, we also manipulated the cue-target SOA on two levels: $150 \mathrm{~ms}$ and $405 \mathrm{~ms}$. At the short SOA, saccades to the cued location would be unhelpful, as the entire cue-target sequence took place in under $200 \mathrm{~ms}$. Any advantage of having time to saccade or move attention to the target should show up as an increased validity effect in the long SOA condition. Varying these five variables orthogonally led to a total of 32 trials in the first block.

The second block consisted solely of line motion and apparent motion trials, randomly intermixed. Number of cues and SOA were manipulated orthogonally. The single-cue trials were included so that at least some trials would generate a clear impression of motion biased in one direction. Half of the 32 trials were line-motion trials, and half were apparent motion trials. This block was included to give us a measure of participants' motion percepts without any potential interference from the letter task.

The third block was a random mixture of the letter discrimination, line-motion, and apparent motion trials used in the first two blocks. This block was included to yield a measure in which participants did not know from trial to trial whether a letter discrimination or a motion judgment would be required of them, so that their attentional state would be the same in both types of trials.

The fourth block was similar to the first, except that on $25 \%$ of trials (16 of 64), the target letter appeared in the invalid location. The invalid trials were randomly intermixed with the valid trials, with the constraint that an equal number of invalid trials appeared in the first and second halves of the block.

Across all four blocks, valid cue position (left or right) was counterbalanced. Practice trials consisted of eight letter discrimination trials (all valid), eight apparent motion trials, and eight line-motion trials.

Procedure. Participants initiated each trial with the space bar. A fixation cross was presented for $1 \mathrm{~s}$, followed by the cue or cues and either two letters (one on each side) or the line, or a central dot. In the short SOA condition, the cues were displayed for $150 \mathrm{~ms}$. In the long SOA condition, the cues remained on for $405 \mathrm{~ms}$ before the letters, or line or dot, were presented, for $45 \mathrm{~ms}$. The cues in both conditions were removed immediately before the letters, line, or dots appeared.

For the letter discrimination task, participants were instructed to respond as quickly and accurately as possible by pressing one of two marked keys on the keyboard, indicating whether a $\mathrm{T}$ or an $\mathrm{X}$ was presented on that trial. Participants responded to line-motion and apparent motion trials with a two-part judgment. The first discrimination was between no motion, lateral motion (to one side only), and inward motion (from both sides). For trials on which motion was seen, participants made more refined judgments. Lateral motion was rated on an 8-point scale ranging from extreme leftward motion to extreme rightward motion. Inward motion was rated on whether it was stronger from one side than the other, on a similar graded scale ranging from mostly leftward motion, through exactly centered motion, to mostly rightward motion. For half of the participants, green was the valid cue color, and for the other half it was red.

\section{Results}

To assess performance on the letter discrimination task, we entered cue validity, SOA, and number of cues into an ANOVA. The reaction times for this analysis were taken from the final block of the experiment. No effects involving the SOA manipulation were reliable (all $p s>.17$ ). This suggests that eye movements alone could not explain any validity effects, as the displays in the short SOA condition were too rapid for any eye movements to be made. The data were therefore collapsed across the two SOA conditions. Participants responded reliably faster with a valid cue than with an invalid cue: Mean reaction times for valid and invalid cues, respectively, were $533 \mathrm{~ms}$ versus $658 \mathrm{~ms}$ for one cue (valid only) and $539 \mathrm{~ms}$ versus $800 \mathrm{~ms}$ for two cues, $F(1,7)=19.0, p<.01$. There was also a significant interaction of cue validity and number of cues, $F(1,7)=$ $9.2, p<.01$. The size of the validity effect was larger for the two-cue condition $(261 \mathrm{~ms})$ than for the one-cue condition $(125 \mathrm{~ms}) .^{3}$

The motion ratings of primary interest are those from the double-cued displays. Inward motion was by far the most common percept reported for these displays $(85 \%$ of trials overall; 7 of 8 participants reported inward motion on more than $66 \%$ of trials). To determine whether having dual tasks affected reported percepts, we compared the mean motion ratings for dot and line trials in Block 2, where these were the only trials, with those for Block 3 , where they were mixed with letter discrimination trials. In Block 2, the mean rating for line motion was -0.24 , and the mean rating for apparent motion was -0.05 . In Block 3 , the mean rating for line motion was 0.03 , and the mean rating for apparent motion was 0.12 . In each condition $38 \%$ of participants had a positive mean rating. An ANOVA showed no difference between the two blocks, $F(1,7)=1.2, n s$. Combined across the two blocks, the mean ratings for both dot $(M=0.04)$ and line trials $(M=-0.11)$ were not significantly different from zero, both $t \mathrm{~s}(7)<1$.

Combining the results from the letter discrimination task and the motion judgment tasks, we have shown that in spite of evidence that participants were attending as instructed, they were no more likely to see line motion or apparent motion from the attended location than from the unattended location. It seems that attention, at least in the sense measured by our letter discrimination task, is not sufficient to produce the line-motion illusion.

We remain puzzled as to the source of the discrepancy between our results and those of Hikosaka et al. (1993b). One difference between our procedure and theirs is that we used untrained, naive observers, whereas their data were taken from a few highly trained observers. It may be that the subjective nature of the illusion makes it, under some circumstances, susceptible to prior expectations. Another difference is that Hikosaka et al. (1993b) used a twoalternative forced-choice response, in which motion is assumed and the observer notes only its direction. These conditions allow even the slightest biases to determine the choice of direction.

\footnotetext{
${ }^{3}$ The validity effect here is large because participants were not aware of the change in validity in the final block. Pilot studies in which participants were informed showed the same pattern of results on both the line motion and letter tasks.
} 


\section{General Discussion}

We have gathered evidence from a variety of sources suggesting that the line-motion illusion is the result of an impletion process that binds successive events to represent a single object. Experiment 1 addressed exogenously directed attention. Postcues produced an illusory shrinking motion, similar phenomenologically to the original illusion, in spite of evidence that attention speeds perception of visual offsets. The effects of dual cues on an intermediate line depend on the presence of an additional outer line, counter to the predictions of a bottom-up, facilitatory model. Finally, temporal and spatial separation between stimuli were shown to have similar effects on apparent motion and illusory line motion.

Manipulations of endogenously directed attention showed a clear dissociation between attention effects facilitating a letter discrimination and the strength or presence of the line-motion illusion, casting doubt on the claim that a gradient of attention plays a causal role in the illusion. In Experiment 2, attention produced a small but reliable linemotion illusion after rotation, but the same conditions also produced a small but reliable perception of apparent motion, of similar strength. In Experiment 3, in spite of demonstrable effects of attention on a discrimination task, neither illusory line motion nor apparent motion was observed.

There are a number of reasons why attention might act differently in the two experiments on both the perceived line motion and apparent motion. One explanation is that attention was initially drawn exogenously to the cue in Experiment 2, whereas cueing could only be endogenous in Experiment 3 . The main point of the two experiments was not to explore the role of attention in apparent motion but rather to demonstrate that where attention does play a role in illusory line motion, it is through the mediating role of binding mechanisms that group visual events into perceived objects in apparent motion. We emphasize that our claim is not that attention has no role whatsoever in the perception of these displays. Attention and the binding mechanisms underlying apparent motion are known to be associated. For example, search times for targets defined by long-range apparent motion increase as a function of the number of distractors, suggesting serial search (Dick, Ullman, \& Sagi, 1987; Horowitz \& Treisman, 1994; Ivry \& Cohen, 1990). In contrast, targets defined by short-range motion exhibit parallel search slopes, suggesting the operation of preattentive motion detectors. Horowitz and Treisman (1994) interpreted these data in terms of feature integration theory (Treisman \& Gelade, 1980), proposing that displays containing multiple sets of elements in long-range apparent motion present the visual system with a binding problem, in which successive elements in neighboring locations must be linked via attention and associated with a single "object file." We propose a similar account of our finding in Experiment $2 \mathrm{~B}$, that endogenously directed attention guides apparent motion in ambiguous situations.

Another study illustrating a link between attention and apparent motion was reported by Stelmach, Herdman, and McNeil (1994). Stelmach et al. presented two stimuli a few degrees apart and asked participants to report whether they saw apparent motion and if so, in which direction. They found that apparent motion was more often perceived to the left when participants attended to the right location and was more often perceived to the right when participants attended to the left location. In displays in which the unattended stimulus preceded the attended stimulus by roughly $50 \mathrm{~ms}$, the direction of apparent motion was ambiguous. In Stelmach et al.'s experiment, attention biases the perceived temporal order of two stimuli, the "prior entry" effect described by Titchener (1908). In our account, the attended element takes precedence in initiating an object file, and the unattended one is subsequently bound to the same object file, giving rise to the perception of apparent motion. Our account does not assume that spatial attention affects the input to peripheral motion detectors.

In sum, attention, in our account, plays two roles in the line-motion displays: (a) It acts as a mechanism for feature binding in multielement displays, and (b) it can bias which sensory data are given precedence in initiating a representation of a single object. In neither case would it generate apparent motion within a physically unitary object like the line used in the displays of Hikosaka et al. (1993b). The motion they describe as occurring within the object, we argue, is actually a progressive transformation or change of shape induced by the binding of the dot and the line as successive states of one moving object. The facts that the line shrinks when the order of cue and line is reversed and that the directional motion is changed by the addition of a second line in our Ternus display (Ternus, 1938), provide strong support for the transformational account of the motion, as does the fact that line motion and apparent motion are modulated in the same way by various stimulus parameters.

Hikosaka et al. (1993a, p. 1237) considered the relationship between apparent motion and the line-motion illusion. We address their arguments in turn:

\section{Apparent Motion Occurs Between Two Stimuli, Not Within One Stimulus}

The motion perceived within the line is not in itself apparent motion in the classical sense; rather, it is a byproduct of an apparent motion process that links the cue and the target into a single perceptual object. The illusory growth of the line is a perceptual transformation that fills in what logically must have occurred over the history of that object.

\section{The Line Illusion Occurs When the Cue and the Line Do Not Strictly Alternate}

Hikosaka et al. (1993a) showed that offset cues, as well as onset cues, produce the illusion. In their displays, two cues were presented, then one was extinguished before the onset of the line. Note that these conditions are even more favorable for apparent motion than those using an onset cue: The first stimulus appears, then disappears before the second 
appears. This sequence is consistent with a single object appearing and jumping to a new location. Perhaps more troublesome is the case in which the cue remains throughout the duration of the line. However, we showed with Experiment $1 \mathrm{C}$ that apparent motion is seen by naive participants under the same conditions. Although it is generally believed that when the first stimulus lasts relatively long, it is the interstimulus interval that determines the quality of perceived motion, our results suggest that the elapsed time between the two onsets is important as well. In the real world, a comparable stimulus could occur if one object was occluding another identical one and then moved to a new location, unmasking the previously occluded object.

\section{Voluntary Allocation of Attention Also Produces the Line-Motion Illusion}

We tested this claim in Experiments 2 and 3, using an additional task to measure the deployment of attention, and found clear dissociations between attention and the linemotion illusion. In Experiment 2, the attentional validity effect was unchanged by rotation, but line motion and apparent motion were greatly reduced. In Experiment 3, despite a strong attentional validity effect produced by endogenous cues, our participants reported neither line motion nor apparent motion. Shimojo et al. (1992) have reported that brief, lateral auditory cues will also elicit illusory line motion. ${ }^{4}$ One possibility is that this effect is also produced by apparent motion. There have been reports of apparent motion between auditory and visual stimuli (e.g., Zapparoli \& Reatto, 1969). Additionally, we suggest that it would be prudent to avoid the use of the forced-choice procedure, which assumes the existence of illusory motion and requires the observer to select only its direction. In the absence of any other difference between conditions, the auditory cue may bias the selection process. It may also be advisable to test the deployment of attention with a probe task for which attentional benefits are known, before an attentional account of this finding is accepted. The same points apply to related claims regarding the effects of tactile stimulation on illusory line motion (Shimojo et al., 1992).

\section{The Illusion Persists When Cue, Target, and Background Are Isoluminant Colors}

Cavanagh, Arguin, and von Grünau (1989) found apparent motion between surfaces defined against the background on any of a number of stimulus dimensions, including isoluminant colors. We suggest that the binding process that associates the cue and line as a single object operates after surfaces have been constructed, regardless of the particular media that define those surfaces.

Another potential criticism of our account is that line motion occurs even when the cue and the target overlap in spatial location, a condition in which apparent motion would not be expected. This raises an important point. We have emphasized the role of apparent motion in invoking the impletion process, but of course other conditions may also elicit it. If two successive surfaces overlap spatially, this is strong evidence that they belong to the same object and that a perceptual transformation should be inferred to link its two states. Besides apparent motion and spatial contiguity, other processes may serve to bind events to the same perceived objects. Several candidates, related to the gestalt grouping principles, have been suggested by Tse and Cavanagh (1995).

\section{Conclusion}

Why do illusions of plastic transformation in objects occur? We suspect that the impletion processes discussed here are of importance to systems responsible for the planning and execution of action. To intercept a moving object, for example, the organism must be able to predict its trajectory through space, through the various possible transformations and occlusions of the image that the object presents to the retina. By ensuring that one's experience of the visual world is smooth and continuous, the visual system enables one to act coherently even though the input is frequently incomplete. We believe that the line-motion illusion may best be explained through this process of implicit inference.

\footnotetext{
${ }^{4}$ We conducted a pilot study with auditory cues, using naive participants and our usual rating scale, including a zero-motion option, and failed to obtain any reports of motion in the line. However, in the same experiment we also failed to obtain attentional effects of the auditory cue on the letter discrimination task that we used in Experiments 2 and 3, making those results inconclusive.
}

\section{References}

Bundesen, C., Larsen, A., \& Farrell, J. E. (1983). Visual apparent movement: Transformations of size and orientation. Perception, $12,549-558$.

Cavanagh, P., Arguin, M., \& von Grünau, M. (1989). Interattribute apparent motion. Vision Research, 29, 1197-1204.

Dick, M., Ullman, S., \& Sagi, D. (1987). Parallel and serial processes in motion detection. Science, 237, 400-402.

Farrell, J. E., \& Shepard, R. N. (1981). Shape, orientation, and apparent rotational motion. Journal of Experimental Psychology: Human Perception and Performance, 7, 477-486.

Faubert, J., \& von Grünau, M. (1992). Split attention and attribute priming in motion induction. Investigative Ophthalmology and Visual Science, 33, 1139.

Faubert, J., \& von Grünau, M. (1995). The influence of two spatially distinct primers and attribute priming on motion induction. Vision Research, 35, 3119-3130.

Hikosaka, O., Miyauchi, S., \& Shimojo, S. (1993a). Focal visual attention produces illusory temporal order and motion sensation. Vision Research, 33, 1219-1240.

Hikosaka, O., Miyauchi, S., \& Shimojo, S. (1993b). Voluntary and stimulus-induced attention detected as motion sensation. Perception, 22, 517-526.

Horowitz, T., \& Treisman, A. (1994). Attention and apparent motion. Spatial Vision, 8, 193-219.

Hunt, S. M. J. (1994). MacProbe. A Macintosh-based experiment- 
er's workstation for the cognitive sciences. Behavior Research Methods, Instruments, and Computers, 26, 345-351.

Ivry, R., \& Cohen, A. (1990). Dissociation of short- and longrange apparent motion in visual search. Journal of Experimental Psychology: Human Perception and Performance, 16, 317-332.

Kahneman, D., Treisman, A., \& Gibbs, B. (1992). The reviewing of object files: Object-specific integration of information. Cognitive Psychology, 24, 175-219.

Kanizsa, G. (1979). Organization in vision: Essays on Gestalt perception. New York: Praeger.

Kawahara, J., Yokosawa, K., Nishida, S., \& Sato, T. (1995). Illusory line motion in visual search. Investigative Ophthalmology and Visual Science, 36, 373.

Kolers, P. A. (1972). Aspects of motion perception. New York: Pergamon Press.

Kolers, P. A., \& von Grünau, M. (1976). Shape and color in apparent motion. Vision Research, 16, 329-335.

Laberge, D. (1983). Spatial extent of attention to letters and words. Journal of Experimental Psychology: Human Perception and Performance, 9, 371-379.

Miyauchi, S., Hikosaka, O., \& Shimojo, S. (1992). Visual attention field can be assessed by illusory line motion sensation. Investigative Ophthalmology and Visual Science, 33, 1262.

Nakayama, K., \& Mackeben, M. (1989). Sustained and transient components of focal visual attention. Vision Research, 29, 1631-1647.

Orlansky, J. (1940). The effect of similarity and difference in form on apparent movement. Archives of Psychology, 246, 1-85.

Posner, M. I. (1980). Orienting of attention. Quarterly Journal of Experimental Psychology, 32, 3-25.

Shepard, R. N. (1984). Ecological constraints on internal represen- tation: Resonant kinematics of perceiving, imagining, thinking, and dreaming. Psychological Review, 91, 417-447.

Shimojo, S., Miyauchi, S., \& Hikosaka, O. (1992). Visual motion sensation yielded by non-visually driven attention. Investigative Ophthalmology and Visual Science, 33 (Suppl.), 1354.

Stelmach, L., Herdman, C., \& McNeil, R. (1994). Attentional modulation of visual processes in motion perception. Journal of Experimental Psychology: Human Perception and Performance, $20,108-121$.

Ternus, J. (1938). The problem of phenomenal identity. In W. D. Ellis (Eds.), A source book of Gestalt psychology (pp. 149-160). New York: Harcourt, Brace.

Titchener, E. (1908). Lectures on the elementary psychology of feeling and attention. New York: Macmillan.

Treisman, A. M., \& Downing, P. (1997). [Attention to offsets, absences, and occlusions]. Unpublished raw data.

Treisman, A. M., \& Gelade, G. (1980). A feature integration theory of attention. Cognitive Psychology, 12, 97-136.

Tse, P., \& Cavanagh, P. (1995). Line motion occurs after surface parsing. Investigative Ophthalmology and Visual Science, 36, 417.

Yantis, S., \& Jonides, J. (1984). Abrupt visual onsets and selective attention: Evidence from visual search. Journal of Experimental Psychology: Human Perception and Performance, 10, 601-620.

Zapparoli, G. C., \& Reatto, L. L. (1969). The apparent movement between visual and acoustic stimulus and the problem of intermodal relations. Acta Psychologica, 29, 256-267.

Received November 2, 1994

Revision received December 15, 1995

Accepted February 12, 1996 